\title{
Comparison of RNAi Sequences in Insect-Resistant Plants to Expressed Sequences of a Beneficial Lady Beetle: A Closer Look at Off-Target Considerations
}

\author{
Margaret L. Allen \\ Biological Control of Pests Research Unit, Agricultural Research Service, United States Department of \\ Agriculture, Stoneville, MS 38776, USA; meg.allen@ars.usda.gov; Tel.: +1-662-686-3647 \\ Academic Editor: Brian T. Forschler \\ Received: 21 October 2016; Accepted: 22 February 2017; Published: 1 March 2017
}

\begin{abstract}
Sequences obtained from transcriptomes of the lady beetle Coleomegilla maculata were compared to those designed for incorporation into crops. Searches of the transcriptomes identified sequences as the most likely to be closely similar to the sequences described in RNAi plant incorporated products. Some proposed prime RNAi pest management targets were also used to identify predicted orthologs from C. maculata. The lady beetle sequences were aligned with sequences from corn rootworms and Colorado potato beetles and, as appropriate in the case of targets, regions of similarity were compared with the genetic model organism for beetles, Tribolium castaneum. Some high levels of nucleotide identity were identified, particularly with an actin-derived sequence from Colorado potato beetle. This actin-derived sequence shared identical sequences with the lady beetle and a parasitic wasp.
\end{abstract}

Keywords: risk assessment; beneficial organism; genetic pest control; lady beetle

\section{Introduction}

One of the most promising emerging insect pest control technologies is based on molecular genetics, and is called RNA interference (RNAi). RNAi is a molecular mechanism that disrupts genes in a target insect prior to the construction of a critical protein, resulting in death of the insect. Double stranded RNA (dsRNA) designed to induce gene knock-down by RNAi in pest insects has been successfully demonstrated and is being developed for implementation in crop protection strategies [1]. RNAi was demonstrated targeting key beetle pests of maize, Zea mays L. (corn), the corn rootworms, and a devastating pest of potatoes, Solanum tuberosum L. the Colorado potato beetle (CBP), Leptinotarsa decemlineata Say (Coleoptera: Chrysomelidae) [2]. Specifically, for field implementation, a sequence from a gene in the genome of the western corn rootworm, Diabrotica virgifera virgifera LeConte (Coleoptera: Chrysomelidae) was incorporated into genetically modified maize to combat the damage incurred by the larvae of this beetle and its close relatives to the roots of maize [3-6]. Another insect targeted for practical RNAi strategies was the Colorado potato beetle (CBP) [7,8]. The potential of RNAi as a tool for pest control is enormous because there are numerous pest targets with many critical genes that could be used in a crop protection, or other pest control, context. The critical genes of the target pest are very likely to be unique to the target because of the degenerate nature of the DNA coding sequence and the variation of genes between organisms. Therefore, a RNAi pest control strategy can be designed that is toxic only to the target insect.

On the other hand, some genes that are crucial to life are highly conserved, or very similar to one another. Genes that are vital to cellular structure and organization, often called "housekeeping genes" may be highly conserved between life forms. This conservation of genes has provided immense benefit to the field of genetics and medicine, because it allows scientists to study genetic mechanisms in one 
organism, a model organism such as a mouse, a fly, a nematode, or a yeast, and predict mechanisms in humans or other non-model life forms. But if a gene has sufficient similarity at the nucleotide (nt) level, there exists the possibility of cross-species or non-target toxicity or other detrimental effect when RNAi is implemented for pest control. And while the quantity and availability of genomic sequencing data are increasing exponentially, beneficial organisms are infrequently sequenced. Thus, gene sequence-level comparisons for the purpose of predicting off-target effects are mostly unavailable.

The lady beetle Coleomegilla maculata De Geer (Coleoptera: Coccinellidae) is a beneficial predator that feeds on the eggs of Colorado potato beetles [9] and also feeds on the pollen of maize [10] and is therefore specifically likely to come into direct contact with the RNAi applications associated with potatoes and maize. A transcriptome analysis of C. maculata was prepared in order to identify differences in gene expression based on adult utilization of foods: diets of pollen compared with insect eggs [11]. A primary rationale for selection of a highly inbred population and performing a sequence analysis on this insect was to establish a genetic foundation for further sequencing, with the aim of contributing to a fully sequenced genome of a representative non-target organism. The pair of transcriptomes were not annotated, and therefore could not be easily utilized for non-target analyses in silico when the RNAi products for maize and potato were in development.

This work describes direct comparison of some sequences obtained from the C. maculata transcriptomes to those designed for incorporation into crops [12]. Searches of the transcriptomes identified sequences as the most likely to be orthologous to the sequences described in RNAi plant incorporated products. Additionally, eleven proposed prime RNAi pest management targets [13] were used to search for similar sequences in the transcriptomes. The identified $C$. maculata sequences were aligned with sequences from corn rootworms and CPB as appropriate, and in the case of targets, regions of similarity were compared with the genetic model organism for beetles, Tribolium castaneum Herbst (Coleoptera: Tenebrionidae).

\section{Materials and Methods}

\section{Identification of C. maculata Sequences and Comparisons}

Transcriptomes of two individual adult insects that were fed two different diets as adults were sequenced and assembled into contigs between 201 base pairs (bp) and >26,000 bp in length [11]. These assembled contigs were compared to National Center for Biological Information (NCBI) Reference RNA sequences (refseq_RNA) [14] and transcriptome shotgun assemblies (TSA) in GenBank using translated BLAST (tBLASTx) algorithm [15]. The spreadsheet generated was sorted and searched to identify sequences that were potentially homologous or orthologous to those evaluated for RNAi pest control sequences targeting D. v. virgifera [12] and L. decemlineata [7], insects in the same order as C. maculata, Coleoptera, and likely to be present in the same North American agroecosystems, maize and potato. Similarly, sequences designated as prime RNAi targets [13] were compared with the paired C. maculata transcriptomes after matching the T. castaneum sequences to GenBank reference mRNA/cDNA sequences. Candidate C. maculata sequences from each assembly were aligned to one another to verify their identity using the NCBI BLAST comparison of two nucleotides setting. Identical or nearly identical sequences were consolidated and reverse transcribed using online sequence manipulation suite (SMS) [16]. Sequences were then loaded into DNAStar EditSeq and aligned using DNAStar MegAlign (Version 12.0. DNASTAR, Madison, WI, USA). The identical portions of the sequences were saved and checked for protein coding translations using the ExPASy Swedish Institute of Bioinformatics (SIB) bioinformatics resource portal translate tool [17]. The translated sequences were compared to characterized genes using the portal's protein BLAST tool.

\section{Results}

A single $C$. maculata sequence with very high similarity to the translated $D$. $v$. virgifera sequence encoding Snf7 was found in each of the two transcriptomes. The two putative C. maculata Snf7 
sequences (CmacSnf7) were identical for $1108 \mathrm{nt}$ and encoded a predicted full length protein of 219 amino acids (aa). The translated sequence is shown in Figure 1A. The predicted CmacSnf7 was aligned to the $240 \mathrm{nt}$ used to develop insect resistant transgenic maize. The sequences and alignments of both nt and aa sequences are shown in Figure 1B. The aa identity was 55/80 or $69 \%$ and the nt identity was $188 / 254$ or $74 \%$. The nt alignment did not result in any continuous nt identities of $>17 \mathrm{nt}$, the expected length for predicting possible off-target effects $[13,18]$.

A single $C$. maculata sequence with very high similarity to the translated sequence encoding the lethal actin dsRNA $A C T$ from L. decemlineata was found in each of the two transcriptomes. The two putative $C$. maculata sequences were identical in nucleotide sequence for $1505 \mathrm{nt}$. The translation of the two sequences identified a predicted full length protein of 376 aa (Figure 2A). The predicted C. maculata actin sequence (CmacAct) was aligned with the $297 \mathrm{nt}$ used to develop transgenic insect resistant potato plants. The sequences and alignments of nt sequences are shown in Figure 2B. The aa identity was $100 \%$ and the nt identity was $257 / 296$ or $87 \%$. The nt alignment resulted in four continuous nt identities of $17 \mathrm{nt}$ or longer, and these longer regions were adjacent to other identical regions (separated by one non-identical nt) (Figure 2B). There were no identical regions longer than $20 \mathrm{nt}$.

A gtcaattaatccaagaacacctgtcattcttgacagccatctagatcttgtgtctattgtgaattgtttaattatattttcag aataatttttgatcattctgaccatattgtttattcaccctctcgaaa ATG AGT TTC TTC TCT AAG ATA TT $\begin{array}{lllllllllll}M & S & F & F & S & K & I & F\end{array}$

GGT GGC AAA AAG GAT GAA GCA CCT AGT ACA GGA GCT GCT ATC CAA AAA CTT CGG GAA ACT GAG

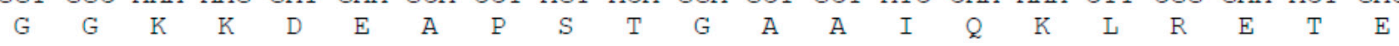

GAG ATG TTA AAT AAG AAA CAA GCA TTT TTG GAA AAG AAA ATA GAG CAA GAA ATT GTC ATT GCA

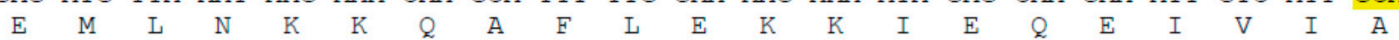

AAA CAA AAC GCT ACT AAG AAT AAG AGA GCT GCT ATT CAG GCC TTG AAA AGG AAG AAG CGA TAT

$\begin{array}{llllllllllllllllllllll}\mathrm{K} & \mathrm{Q} & \mathrm{N} & \mathrm{A} & \mathrm{T} & \mathrm{K} & \mathrm{N} & \mathrm{K} & \mathrm{R} & \mathrm{A} & \mathrm{A} & \mathrm{I} & \mathrm{Q} & \mathrm{A} & \mathrm{L} & \mathrm{K} & \mathrm{R} & \mathrm{K} & \mathrm{K} & \mathrm{R} & \mathrm{Y}\end{array}$

GAA AAG CAA CTC CAA CAA ATT GAT GGC ACC CTC ACT ACT TTA GAG TTA CAA AGA GGA ACA TTA

$\begin{array}{lllllllllllllllllllll}\mathrm{E} & \mathrm{K} & \mathrm{Q} & \mathrm{L} & \mathrm{Q} & \mathrm{Q} & \mathrm{I} & \mathrm{D} & \mathrm{G} & \mathrm{T} & \mathrm{L} & \mathrm{T} & \mathrm{T} & \mathrm{L} & \mathrm{E} & \mathrm{L} & \mathrm{Q} & \mathrm{R} & \mathrm{G} & \mathrm{T} & \mathrm{L}\end{array}$

GAG GAA GCT GTA ACA AAT ACT GAT GTC ATT CAA ACA ATG AAA GAT GCT GCG GAT GCA ATT AAG

$\begin{array}{lllllllllllllllllllll}\mathrm{E} & \mathrm{E} & \mathrm{A} & \mathrm{V} & \mathrm{T} & \mathrm{N} & \mathrm{T} & \mathrm{D} & \mathrm{V} & \mathrm{I} & \mathrm{Q} & \mathrm{T} & \mathrm{M} & \mathrm{K} & \mathrm{D} & \mathrm{A} & \mathrm{A} & \mathrm{D} & \mathrm{A} & \mathrm{I} & \mathrm{K}\end{array}$

CAC GCT CAT AAA CAT ATG AAT GTT GAT CAA GTG CAC GAT ATA ATG GAT GAT ATA GCT GAG CAA

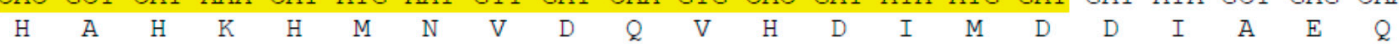

CAA GAT GTA GCT AAT GAA ATA TCA CAA GCT ATT AGC AAT CCT ATT GGT TTC GGT GAA GAT ATT

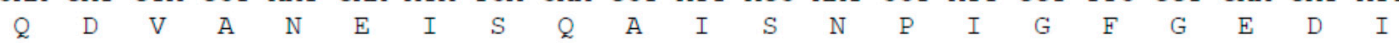

GAT GAA GAT GAA TTG AAC AAG GAA CTT GAA GAC CTC GAA CAA GAA ACA TTA GAT AGT GAA TTA

$\begin{array}{lllllllllllllllllllllllllll}D & \mathrm{E} & \mathrm{D} & \mathrm{E} & \mathrm{L} & \mathrm{N} & \mathrm{K} & \mathrm{E} & \mathrm{L} & \mathrm{E} & \mathrm{D} & \mathrm{L} & \mathrm{E} & \mathrm{Q} & \mathrm{E} & \mathrm{T} & \mathrm{L} & \mathrm{D} & \mathrm{S} & \mathrm{E} & \mathrm{L}\end{array}$

CTC GAT ATC ACT TTA CCT GCC GAT AAA CTT CCT GAT GTA CCT AAA GAA GCT GTG AAA CCC AAA

$\begin{array}{lllllllllllllllllllllll}\mathrm{L} & \mathrm{D} & \mathrm{I} & \mathrm{T} & \mathrm{L} & \mathrm{P} & \mathrm{A} & \mathrm{D} & \mathrm{K} & \mathrm{L} & \mathrm{P} & \mathrm{D} & \mathrm{V} & \mathrm{P} & \mathrm{K} & \mathrm{E} & \mathrm{A} & \mathrm{V} & \mathrm{K} & \mathrm{P} & \mathrm{K}\end{array}$

CCA ACT TCA TCC AAA AAA GCT GTT GAA GAT GAT GAG GAT ATG AAG GCC TTA GCA GAA TGG GCA

$\begin{array}{lllllllllllllllllllll}\mathrm{P} & \mathrm{T} & \mathrm{S} & \mathrm{S} & \mathrm{K} & \mathrm{K} & \mathrm{A} & \mathrm{V} & \mathrm{E} & \mathrm{D} & \mathrm{D} & \mathrm{E} & \mathrm{D} & \mathrm{M} & \mathrm{K} & \mathrm{A} & \mathrm{L} & \mathrm{A} & \mathrm{E} & \mathrm{W} & \mathrm{A}\end{array}$

TCA TAA gagcagaagtgtcttaaaatccacttagtggtttccttgactaaccgtacttgtcaagtggtatcttattaggg

S stop

atttcgcctctgattgaaaataaattgcttraacttgtaaatatgatgtacacattgagaaaaactacaaaatattcgaaaact tggaattatatatcacaatgaaaaacatgaattgaaagtttctgatagttgtcaaatattattcattattctaaaaagatggct tttgtaatggttaggagttcattaatattttgctaactcataattatttgattatatgttgaagatct

Figure 1. Cont. 
B. 240 nt Monsanto Snf7 sequence from Diabrotica virgifera virgifera:

GCA AAG AAA AAT GCG TCG AAA AAT AAA AGA GTT GCA CTC CAA GCC CTC AAA AAG AAG AAA CGA

TTG GAA AAG ACC CAA CTA CAA ATA GAT GGA ACC CTT ACA ACT ATT GAA ATG CAG AGG GAA GCC

CTC GAA GGA GCT AGC ACA AAT ACT GCT GTA TTA GAT TCT ATG AAA AAT GCT GCA GAT GCC CTT

AAG AAA GCT CAT AAG AAT TTG AAT GTA GAT GAT GTT CAC GAT ATC ATG GAT

240 nt from Coleomegilla maculata predicted homolog:

GCA AAA CAA AAC GCT ACT AAG AAT AAG AGA GCT GCT ATT CAG GCC TTG AAA AGG AAG AAG CGA

TAT GAA AAG CAA CTC CAA CAA ATT GAT GGC ACC CTC ACT ACT TTA GAG TTA CAA AGA GGA ACA

TTA GAG GAA GCT GTA ACA AAT ACT GAT GTC ATT CAA ACA ATG AAA GAT GCT GCG GAT GCA ATT

AAG CAC GCT CAT AAA CAT ATG AAT GTT GAT CAA GTG CAC GAT ATA ATG GAT

D. v. virgifera GCA AAG AAA AAT GCG TCG AAA AAT AAA AGA GTT GCA CTC CAA GCC CTC AAA

C. II| || || || || | || ||| || ||| | | || | | || ||| | ||

c. maculata GCA AAA CAA AAC GCT ACT AAG AAT AAG AGA GCT GCT ATT CAG GCC TTG AAA

D. v. virgifera AAG AAG AAA CGA TTG GAA AAG ACC CAA CTA CAA ATA GAT GGA ACC CTT ACA

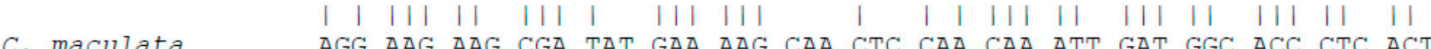

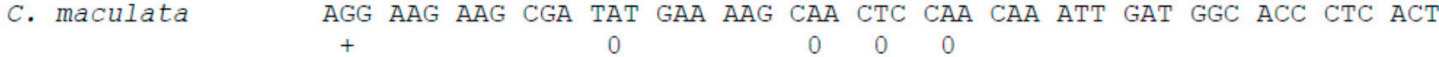

D. v. virgifera ACT ATT GAA ATG CAG AGG GAA GCC CTC GAA GGA GCT AGC ACA AAT ACT GCT

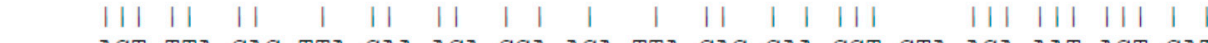

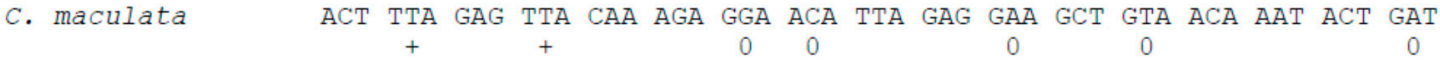

D. v. virgifera GTA TTA GAT TCT ATG AAA AAT GCT GCA GAT GCC CTT AAG AAA GCT CAT AAG

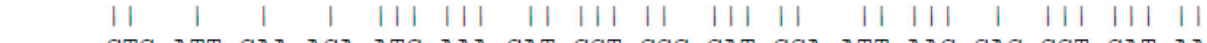

c. maculata GTC ATT CAA ACA ATG AAA GAT GCT GCG GAT GCA ATT AAG CAC GCT CAT AAA

D. v. virgifera AAT TTG AAT GTA GAT GAT GTT CAC GAT ATC ATG GAT

C. maculata

+
+

188/254 nt identities(74\%) with $28 / 254$ gaps (11\%).

D. V. virgifera

AKKNASKNKRVALQALKKKKRLEKTQLQIDGTLTTIEMQREALEGASTNTAVLDSMKNAA

C. maculata $A K+N A+K N K R \quad A+Q A L K+K K R \quad E K \quad Q I D G T L T T+E+Q R$ LE A TNT $V++M K+A A$

D. v. virgifer AKQNATKNKRAAIQALKRKKRYEKQLQQIDGTLTTLELQRGTLEEAVTNTDVIQTMKDAA

C. maculata

DALKKAHKNLNVDDVHDIMD

$\mathrm{DA}+\mathrm{K} \quad \mathrm{AHK}++\mathrm{NVD}$ VHDIMD

DAIKHAHKHMNVDQVHDIMD

$55 / 80$ aa identities (69\%) with $67 / 80$ similarities (83\%) and no gaps.

Figure 1. Predicted sequence and translation from Coleomegilla maculata transcriptomes most similar to Diabrotica virgifera virgifera Snf7 used to design rootworm resistant maize. (A) Complete cDNA sequence. Highlighted section is the portion of the sequence matching the maize RNAi transgene. Upper case indicates translated sequence, lower case untranslated; (B) Alignment of the RNAi transgene regions. Lines between nucleotides indicate identity, identical letters between amino acid sequences indicate identity. The symbol (+) indicates an amino acid substitution by a similar residue, while a (0) or a blank between nucleotide or amino acid letter, respectively, indicates a non-similar substitution. Bottom: amino acid alignment. 
A. gtattcagtcagtcgccaagctattcagcttcgagttctttctgtctgtgcatctgtgtaacgtaaattagacatc ATG TGT GAC GAC GAT GTT GCG GCT CTT GTC GTT GAC AAT GGT TCC GGT ATG TGC AAG GCC GGT TTC $\begin{array}{llllllllllllllllllllll}M & \text { C } & \text { D } & \text { D } & \text { D } & \text { V } & \text { A } & \text { A } & \text { L } & \text { V } & \text { V } & \text { D } & \text { N } & \text { G } & \text { S } & \text { G } & \text { M } & \text { C } & \text { K } & \text { A } & \text { G } & \text { F }\end{array}$ GCT GGG GAT GAT GCC CCA CGT GCC GTG TTC CCA TCC ATC GTT GGT CGC CCA AGg CAT CAG GGT GTG $\begin{array}{lllllllllllllllllllllll}\text { A } & G & \text { D } & \text { D } & \text { A } & \text { P } & \text { R } & \text { A } & \text { V } & \text { F } & \text { P } & \text { S } & \text { I } & \text { V } & \text { G } & \text { R } & \text { P } & \text { R } & \text { H } & \text { \& } & G & \text { V }\end{array}$ ATG GTT GGT ATG GGA CAA AAA GAC TCA TAT GTA GGA GAT GAA GCT CAA AGC AAG AGA GGT ATT CTC ACC TTG AAA TAC CCC ATC GAA CAT GGA ATC ATC ACC AAC TGG GAT GAT ATG GAA AAA ATC TGG CAC CAC ACC TTC TAC AAC GAA CTC CGT GTA GCA CCA GAA GAA CAC CCT GTC CTT TTG ACT GAA GCT CCA $\begin{array}{lllllllllllllllllllllll}\mathrm{H} & \mathrm{T} & \mathrm{F} & \mathrm{Y} & \mathrm{N} & \mathrm{E} & \mathrm{L} & \mathrm{R} & \mathrm{V} & \mathrm{A} & \mathrm{P} & \mathrm{E} & \mathrm{E} & \mathrm{H} & \mathrm{P} & \mathrm{V} & \mathrm{L} & \mathrm{L} & \mathrm{T} & \mathrm{E} & \mathrm{A} & \mathrm{P}\end{array}$ TTG AAC CCA AAA GCT AAC AGA GAA AAA ATG ACC CAA ATC ATG TTT GAG ACC TTT AAC ACA CCA GCT $\begin{array}{llllllllllllllllllllll}\mathrm{L} & \mathrm{N} & \mathrm{P} & \mathrm{K} & \mathrm{A} & \mathrm{N} & \mathrm{R} & \mathrm{E} & \mathrm{K} & \mathrm{M} & \mathrm{T} & \mathrm{Q} & \mathrm{I} & \mathrm{M} & \mathrm{F} & \mathrm{E} & \mathrm{T} & \mathrm{F} & \mathrm{N} & \mathrm{T} & \mathrm{P} & \mathrm{A}\end{array}$ ATG TAT GTC GCC ATC CAA GCT GTA CTA TCT TTG TAT GCC TCT GGT CGT ACC ACC GGT ATC GTT TTG $\begin{array}{llllllllllllllllllllll}M & Y & V & A & I & Q & A & \text { V } & \text { L } & \text { S } & \text { L } & \text { Y } & \text { A } & \text { S } & G & \text { R } & \text { T } & \text { T } & \text { G } & \text { I } & \text { V } & \text { L }\end{array}$ GAC TCA GGA GAT GGT GTA TCT CAC ACT GTA CCA ATC TAT GAA GGT TAC GCC CTT CCT CAC GCC ATC $\begin{array}{lllllllllllllllllllllll}D & S & G & D & G & V & \text { S } & \text { H } & \text { T } & \text { V } & \text { P } & \text { I } & \text { Y } & \text { E } & \text { G } & \text { Y } & \text { A } & \text { L } & \text { P } & \text { H } & \text { A } & \text { I }\end{array}$

CTC CGT CTT GAC TTG GCT GGT CGT GAC TTG ACC GAC TAC CTT Atg AAA ATC CTC ACC GAA AGg GGT $\begin{array}{lllllllllllllllllllllll}\mathrm{L} & \mathrm{R} & \mathrm{L} & \mathrm{D} & \mathrm{L} & \mathrm{A} & \mathrm{G} & \mathrm{R} & \mathrm{D} & \mathrm{L} & \mathrm{T} & \mathrm{D} & \mathrm{Y} & \mathrm{L} & \mathrm{M} & \mathrm{K} & \mathrm{I} & \mathrm{L} & \mathrm{T} & \mathrm{E} & \mathrm{R} & \mathrm{G}\end{array}$

TAC TCA TTC ACC ACC ACC GCT GAG AGG GAA ATC GTT CGT GAC ATC AAG GAG AAA CTT TGC tAt GTC $\begin{array}{lllllllllllllllllllllll}\mathrm{Y} & \mathrm{S} & \mathrm{F} & \mathrm{T} & \mathrm{T} & \mathrm{T} & \mathrm{A} & \mathrm{E} & \mathrm{R} & \mathrm{E} & \mathrm{I} & \mathrm{V} & \mathrm{R} & \mathrm{D} & \mathrm{I} & \mathrm{K} & \mathrm{E} & \mathrm{K} & \mathrm{I} & \mathrm{C} & \mathrm{Y} & \mathrm{V}\end{array}$ GCC CTC GAC TTC GAA CAG GAA ATG GCC ACC GCC GCT GCT TCC ACC TCA TTG GAG AAA TCC TAT GAA

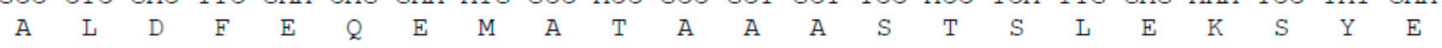
CTT CCC GAC GGT CAA GTT ATC ACC ATC GGT AAC GAA AGA TTC CGT TGC CCT GAA GCC CTC TTC CAA $\begin{array}{llllllllllllllllllllll}I & P & D & G & Q & V & I & T & I & G & N & E & R & F & R & C & P & E & A & L & F & Q\end{array}$ CCT TCC TTC TTG GGT ATG GAA TCC TGT GGT ATT CAT GAA ACT GTC TAC AAC TCC ATC ATG AAG TGT

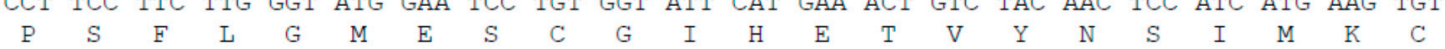
GAC GTC GAT ATC CGT AAG GAC TTG TAC GCC AAC ACC GTA CTC TCT GGT GGT ACC ACC ATG TAC CCC $\begin{array}{lllllllllllllllllllllll}\mathrm{D} & \mathrm{V} & \mathrm{D} & \mathrm{I} & \mathrm{R} & \mathrm{K} & \mathrm{D} & \mathrm{L} & \mathrm{Y} & \mathrm{A} & \mathrm{N} & \mathrm{T} & \mathrm{V} & \mathrm{L} & \mathrm{S} & \mathrm{G} & \mathrm{G} & \mathrm{T} & \mathrm{T} & \mathrm{M} & \mathrm{Y} & \mathrm{P}\end{array}$ GGT ATC GCC GAT AGG ATG CAA AAG GAA ATC ACT GCC TTG GCA CCA TCA ACC ATC AAG ATC AAG ATC $\begin{array}{lllllllllllllllllllllllll}G & I & A & D & R & M & \& & K & E & I & T & A & I & A & P & S & T & I & K & I & K & I\end{array}$ ATT GCT CCC CCA GAA AGG AAA TAC TCT GTC TGG ATC GGA GGA TCC ATC TTG GCT TCC CTA TCC ACC $\begin{array}{lllllllllllllllllllllll}I & A & P & P & E & R & K & Y & S & V & W & I & G & G & S & I & L & A & S & L & S & T\end{array}$ TTC CAA CAG ATG TGG ATC TCC AAA CAA GAA TAC GAC GAA TCC GGC CCT GGA ATT GTC CAC CGC AAA $\begin{array}{lllllllllllllllllllllll} & \& & \& & \& & M & W & I & S & K & Q & E & Y & D & E & S & G & P & G & I & V & H & R & K\end{array}$ TGC TTC TAA gtcaattaattttacatcctttgtcatcatgttgtattgtattatactcaaaaatctttttatagatgcgactt C $\quad$ F stop

tgatgcactgccatcatacagacaagaacaacaatcgaagaactcagttgcaagccaacaaaagcaaaatagcctgactcaattattt caattgcccatttatttaaaatcttataagtttatttatgttatatttattattattcatgtattttttaaataaattcogcaat atttataagtttattgaataaagaattcaaataaaaaaaaaa

Figure 2. Cont. 
B. $297 \mathrm{nt}$ actin sequence from Leptinotarsa decemlineata:

gcacgaggtttttctgtctagtgagcagtgtccaacctcaaagacaa ATGIGTGACGACGATGTAGCGGCTCTTGTCGTAGACAA TGGATCCGGTATGTGCAAAGCCGGTTTCGCAGGAGATGACGCACCCCGTGCCGTCTTCCCCTCGATCGTCGGTCGCCCAAGGCATCA AGGAGTCATGGTCGGTATGGGACAAAAGGACTCATACGTAGGAGATGAAGCCCAAAGCAAAAGAGGTATCCTCACCCTGAAATACCC CATCGAACACGGTATCATCACCAACTGGGATGACAT

296 nt from Coleomegilla maculata predicted actin homolog:

cttcgagttctttctgtctgtgcatctgtgtaacgtaattagacatcATGTGTGACGACGATGTTGCGGCTCTTGTCGTTGACAAT GGTTCCGGTATGTGCAAGGCCGGTTTCGCTGGGGATGATGCCCCACGTGCCGTGTTCCCATCCATCGTTGGTCGCCCAAGGCATCAG GGTGTGATGGTTGGTATGGGACAAAAAGACTCATATGTAGGAGATGAAGCTCAAAGCAAGAGAGGTATTCTCACCTTGAAATACCCC ATCGAACATGGAATCATCACCAACTGGGATGATAT
L. decemlineata
4
cgaggttttctgtctagtg-agcagtgtccaacctcaaa-agacaacATGTGTGACGAC

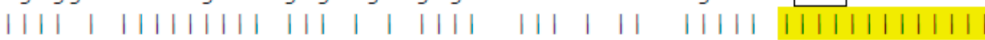
C. maculata
L. decemlineata
C. maculata
I. decemlineata
C. maculata
L. decemlineata
ATGGTCGGTATGGGACAAAAGGACTCATACGTAGGAGATGAAGCCCAAAGCAAAAGAGGT ||||| |||||||||||||| |||||||| |||||||||||||| |||||||| |||||
C. maculata 181 ATGGTTGGTATGGGACAAAAAGACTCATATGTAGGAGATGAAGCTCAAAGCAAGAGAGGT
I. decemlineata
C. maculata

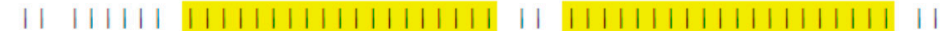
$257 / 296$ nt identities (87\%) with 5/296 gaps (1\%).

Identical translation: MCDDDVAALVVDNGSGMCKAGFAGDDAPRAVFPSIVGRPRHQGVMVGMGQKDSYVGDEAQSKRG ILTLKYPIEHGIITNWDD

Figure 2. Predicted sequence and translation from Coleomegilla maculata transcriptomes most similar to Leptinotarsa decemlineata $\beta$-actin used to design beetle resistant potato. (A) Complete cDNA sequence. Highlighted section is the portion of the sequence matching the potato RNAi transgene. Upper case indicates translated sequence, lower case untranslated. Start sites are underscored in unaligned nt sequences; (B) Alignment of the RNAi transgene regions. Translation start sites are boxed. Lines between nucleotides indicate identity, highlighting indicates continuous regions of nucleotide identity. Translated sequence is not included with nucleotides and translation is shown without alignment because all translated residues are identical.

Eleven sequences from the T. castaneum genome predicted as prime target genes for RNAi disruption [13] were used to seek similar sequences in the $C$. maculata transcriptomes. The search results are summarized in Table 1 ; three of the sequences did not result in a probable match, but the other eight were highly similar to both the aa and nt sequences from unique predicted genes. Each pair of predicted C. maculata sequence encoded at least one full length translation; one sequence predicted four isoforms (the contigs similar to L82/gw, predicted reference sequence XM_015982857). Only one of the eight predicted gene sequences, the one similar to L55/pp1 alpha-96a, predicted reference sequence XM_001813922, did not contain a region of 17 or more contiguous nucleotide identities. 
Table 1. Eleven novel RNAi target genes from Tribolium castaneum compared with Coleomegilla maculata predicted homologue sequences.

\begin{tabular}{|c|c|c|c|c|c|c|c|}
\hline \multirow{2}{*}{ Symbol/Name } & \multirow{2}{*}{ Refseq ID } & \multicolumn{3}{|c|}{ Sequence Identification from $C$. maculata Transcriptomes } & \multicolumn{3}{|c|}{ T. castaneum vs. C. maculata } \\
\hline & & Pollen-Fed & Insect Egg-Fed & Similarity (C. mac) & aa Identities & nt Similarity & 17+ nt Matches \\
\hline $\mathrm{L} 10 /$ cact & NM_001163711 & not found & not found & $\mathrm{n} / \mathrm{a}$ & $\mathrm{n} / \mathrm{a}$ & $\mathrm{n} / \mathrm{a}$ & $\mathrm{n} / \mathrm{a}$ \\
\hline $\mathrm{L} 11 / \operatorname{srp} 54 k$ & XM_962796 & comp3017 & comp3093 & $1558 / 1558$ & $474 / 508$ & $1104 / 1415(78 \%)$ & 2 \\
\hline $\mathrm{L} 44 /$ rop & NM_001170684 & comp12493 & comp11584 & $2442 / 2449$ & $528 / 590$ & $1321 / 1781(74 \%)$ & 4 \\
\hline L47/alpha snap & XM_968056 & comp8167 & comp5787 & $2338 / 2338$ & $212 / 241$ & $651 / 879(74 \%)$ & 1 \\
\hline $\mathrm{L} 50 / \operatorname{sh} i$ & XM_008200600 & not found & not found & $\mathrm{n} / \mathrm{a}$ & $\mathrm{n} / \mathrm{a}$ & $\mathrm{n} / \mathrm{a}$ & $\mathrm{n} / \mathrm{a}$ \\
\hline L55/pp1alpha-96a & XM_001813922 & comp10238 & comp10443 & $1751 / 1752$ & $318 / 327$ & $781 / 986(79 \%)$ & 0 \\
\hline $\mathrm{L} 67 /$ inr-a & XM_008194324 & not found & not found & $\mathrm{n} / \mathrm{a}$ & $\mathrm{n} / \mathrm{a}$ & $\mathrm{n} / \mathrm{a}$ & $\mathrm{n} / \mathrm{a}$ \\
\hline $\mathrm{L} 76 / h s c 70-3$ & XM_008202764 & comp14394 & comp13114 & $2433 / 2488$ & $598 / 645$ & $1528 / 1924(79 \%)$ & $8 / 7$ \\
\hline $\mathrm{L} 80 / r p n 7$ & XM_968550 & comp12477 & comp14743 & $1296 / 1301$ & $322 / 389$ & $849 / 1173(72 \%)$ & 1 \\
\hline $\mathrm{L} 82 / g w$ & XM_015982857 & comp8599 & comp10202 & $4423 / 4424$ & $967 / 1388$ & $1781 / 2609(68 \%)$ & 11 \\
\hline $\mathrm{L} 84 / r p t 3$ & XM_962883 & comp3229 & comp12416 & $1406 / 1434$ & $393 / 409$ & $907 / 1211(75 \%)$ & 1 \\
\hline
\end{tabular}




\section{Discussion}

RNAi was initially described as an intracellular, or cell autonomous, process. It was soon further elucidated as a systemic process within multicellular organisms, and then shown to function through external exposure via feeding or soaking an organism [12]. Introduction of dsRNA to an organism from an external source has been termed "environmental RNAi" (eRNAi) [19]. The potential for eRNAi as a crop protection strategy was recognized and tested in nematodes $[20,21]$ and insects $[2,22]$ soon after demonstration in model organisms. Progression to a practical field implementation against the target insect $D$. v. virgifera followed rapidly $[3,4,6]$, and research progressed to include addressing concerns of impact to non-target organisms. A thorough set of studies to assess risk to non-target arthropods concluded in field tests that the presence of beneficials, identified as predatory earwigs, lacewings, lady beetles, minute pirate bugs, parasitic wasps, and spiders, in statistically similar quantities indicated no risk [23]. Plot sizes designated for the counts were less than $100 \mathrm{~m}^{2}$, and the life stage of arthropods counted was not specified. Studies have also included laboratory bioassays, and C. maculata was one of the laboratory test species for risk assessment. When treated with diet incorporating the Snf7 dsRNA, results conclusively demonstrated no ill effects [3]. For lady beetles, there is wide variation among species in dietary habits. Most are predatory, although the Mexican bean beetle, Epilachna varivestis Mulsant (Coleoptera: Coccinellidae), is a serious pest and feeds on living plant tissue. While some predatory lady beetles specialize on a single prey species, some are more catholic in dietary choices. The North American native species (or species complex) C. maculata is known to consume pollen as a substantial portion of its diet $[10,24]$. This species, a logical choice for non-target testing in North America, was included as one of the non-target arthropods for environmental risk testing. The sequence comparison provided here and shown in Figure 1B provides further demonstration that the dsRNA used for transgenic corn rootworm resistant maize varieties should not interfere with the predicted CmacSnf7 gene. The nt alignment did not result in any continuous nt identities of $>17 \mathrm{nt}$, a predicted minimal length suggested for predicting off-target effects $[13,18]$. In fact, the longest series of nt identities of the sequences was 9 . While predicting off-target effects by searching for $17+$ continuous nt similarity is not a certainty, particularly when considering diverse organisms such as insects, short RNAs as small as $17 \mathrm{nt}$ may produce gene interference in some insects [13].

In a series of innovative experiments, dsRNA constructs were inserted into the genome of potato plant chloroplasts [7]. This strategy increased the likelihood of delivery of the effective long dsRNAs to the target pest, L. decemlineata, and was shown to be highly lethal [7]. A dsRNA similar to the D. v. virgifera sequence $S n f 7, S H R$, was less lethal than a dsRNA from a portion of the sequence from an actin gene [7]. The CmacSnf7 sequence had even less identity to the $220 \mathrm{nt}$ sequence tested in L. decemlineata, SHR, $142 / 212$ or $67 \%$ (compare to Figure 1B). The longest identical nt segment was $12 \mathrm{nt}$ (not shown). However, the actin gene used in the experiments was very similar to the CmacAct gene identified from transcriptomes. While there were no identical regions longer than $21 \mathrm{nt}$, the canonical effective siRNA length, the region between nt 60 and nt 115 has only four mismatches. This similarity warrants further risk assessment studies if the actin gene sequence is further implemented as a crop protection strategy. C. maculata is known to consume the eggs of L. decemlineata, and in a potato agroecosystem it is possible that the pest eggs could constitute substantial portion of the beneficial lady beetles' diet. While it could be argued that eggs have not fed and could not contain plant-derived dsRNA, it could also be possible that a female adult $L$. decemlineat a feeding on dsRNA could transfer some portion of the dsRNA to eggs during oogenesis, exposing the beneficial insect. More disturbingly, the L. decemlineata ACT sequence is closely identical to a sequence on file in GenBank for a species of commercially produced beneficial generalist parasitoid, Trichogramma pretiosum Riley (Hymenoptera: Trichogrammidae), with continuous nt identity regions up to $32 \mathrm{nt}$ long (sequence XM_014379004.1).

For future RNAi pest control development, a robust screen of the genetic model insect $T$. castaneum indicated some categories of genes and eleven specific candidate genes for use [13]. Direct comparison of the eleven candidate genes clearly identified eight predicted orthologs in C. maculata (Table 1). While some of the identified transcriptome sequences were not precisely alike in the two specimen 
assemblies, the variations could be explained by minor sequencing error, different alleles containing nucleotide polymorphisms, or diet-induced expression variation. The three genes that were not found could be present in the C. maculata genome but were not expressed in the adult stage that was used for transcriptome sequencing. The 17+ nt identity occurrences between two distantly related beetles, T. castaneum and C. maculata, suggests that careful analysis of non-target species when choosing target genes is warranted. That being said, the eight predicted potential target genes compared in Table 1 were long enough to provide ample portions of sequence for dsRNA construction while avoiding those sites with non-target nt identity. Sequencing of the genomes or transcriptomes of an increasing number of non-target species should be undertaken to support good decision making. Decisions concerning non-targets for sequencing or bioassays could be assisted by tools such as the database described for use in portions of Europe [25]. An elegant tool designed to compare RNAi targets for one species against other species was developed [26]. However, without available sequences from beneficial and benign species the program has limited utility.

\section{Conclusions}

The analyses performed here, sequence comparisons based on transcriptome data from a limited, highly inbred sample, can neither guarantee biosafety nor prove environmental risk. Nonetheless, the close identity of actin sequences demonstrated by this analysis may serve as an illustration for selection of target genes intended for commercialization. There is an enormous quantity of potential RNAi targets for use in both research and agricultural implementation. The selectivity and specificity of RNAi has great potential, as future research and development will doubtless prove. Further sequencing of non-target organisms will speed up and enhance target gene choice and support risk assessments.

Acknowledgments: An earlier version of this manuscript was reviewed by William Rodney Cooper, C. Doug Boyette, and Michael Grodowitz, and the comments and suggestions provided helpful improvement. Additional suggestions were provided by anonymous reviewers. The United States Government has the right to retain a non-exclusive, royalty-free license in and to any copyright of this article. This article reports the results of research only; mention of a commercial or proprietary product does not constitute an endorsement of the product by the United States Department of Agriculture. USDA is an equal opportunity provider and employer.

Conflicts of Interest: The author declares no conflict of interest.

\section{References}

1. Poelchau, M.F.; Coates, B.S.; Childers, C.P.; Peréz de León, A.A.; Evans, J.D.; Hackett, K.; Shoemaker, D. Agricultural applications of insect ecological genomics. Curr. Opin. Insect Sci. 2016, 13, 61-69. [CrossRef] [PubMed]

2. Baum, J.A.; Bogaert, T.; Clinton, W.; Heck, G.R.; Feldmann, P.; Ilagan, O.; Johnson, S.; Plaetinck, G.; Munyikwa, T.; Pleau, M.; et al. Control of coleopteran insect pests through RNA interference. Nat. Biotechnol. 2007, 25, 1322-1326. [CrossRef] [PubMed]

3. Bachman, P.M.; Bolognesi, R.; Moar, W.J.; Mueller, G.M.; Paradise, M.S.; Ramaseshadri, P.; Tan, J.; Uffman, J.P.; Warren, J.; Wiggins, B.E.; et al. Characterization of the spectrum of insecticidal activity of a double-stranded RNA with targeted activity against western corn rootworm (Diabrotica virgifera virgifera LeConte). Transgenic Res. 2013, 22, 1207-1222. [CrossRef] [PubMed]

4. Bolognesi, R.; Ramaseshadri, P.; Anderson, J.; Bachman, P.; Clinton, W.; Flannagan, R.; Ilagan, O.; Lawrence, C.; Levine, S.; Moar, W.; et al. Characterizing the mechanism of action of double-stranded RNA activity against western corn rootworm (Diabrotica virgifera virgifera LeConte). PLoS ONE 2012, 7, e47534. [CrossRef] [PubMed]

5. Koci, J.; Ramaseshadri, P.; Bolognesi, R.; Segers, G.; Flannagan, R.; Park, Y. Ultrastructural changes caused by Snf7 RNAi in larval enterocytes of western corn rootworm (Diabrotica virgifera virgifera LeConte). PLoS ONE 2014, 9, e83985. [CrossRef] [PubMed] 
6. Ramaseshadri, P.; Segers, G.; Flannagan, R.; Wiggins, E.; Clinton, W.; Ilagan, O.; McNulty, B.; Clark, T.; Bolognesi, R. Physiological and cellular responses caused by RNAi- mediated suppression of Snf7 orthologue in western corn rootworm (Diabrotica virgifera virgifera) larvae. PLoS ONE 2013, 8, e54270. [CrossRef] [PubMed]

7. Zhang, J.; Khan, S.A.; Hasse, C.; Ruf, S.; Heckel, D.G.; Bock, R. Full crop protection from an insect pest by expression of long double-stranded RNAs in plastids. Science 2015, 347, 991-994. [CrossRef] [PubMed]

8. Zhu, F.; Xu, J.J.; Palli, R.; Ferguson, J.; Palli, S.R. Ingested RNA interference for managing the populations of the Colorado potato beetle, Leptinotarsa decemlineata. Pest Manag. Sci. 2011, 67, 175-182. [CrossRef] [PubMed]

9. Mallampalli, N.; Gould, F.; Barbosa, P. Predation of Colorado potato beetle eggs by a polyphagous ladybeetle in the presence of alternate prey: Potential impact on resistance evolution. Entomol. Exp. Appl. 2005, 114, 47-54. [CrossRef]

10. Lundgren, J.G.; Wiedenmann, R.N. Nutritional suitability of corn pollen for the predator Coleomegilla maculata (Coleoptera: Coccinellidae). J. Insect Physiol. 2004, 50, 567-575. [CrossRef] [PubMed]

11. Allen, M.L. Characterization of adult transcriptomes from the omnivorous lady beetle Coleomegilla maculata fed pollen or insect egg diet. J. Genom. 2015, 3, 20-28. [CrossRef] [PubMed]

12. Ivashuta, S.; Zhang, Y.; Wiggins, B.E.; Ramaseshadri, P.; Segers, G.C.; Johnson, S.; Meyer, S.E.; Kerstetter, R.A.; McNulty, B.C.; Bolognesi, R.; et al. Environmental RNAi in herbivorous insects. RNA 2015, 21, 840-850. [CrossRef] [PubMed]

13. Ulrich, J.; Dao, V.A.; Majumdar, U.; Schmitt-Engel, C.; Schwirz, J.; Schultheis, D.; Strohlein, N.; Troelenberg, N.; Grossmann, D.; Richter, T.; et al. Large scale RNAi screen in Tribolium reveals novel target genes for pest control and the proteasome as prime target. BMC Genom. 2015, 16, 1-9. [CrossRef] [PubMed]

14. Pruitt, K.D.; Tatusova, T.; Brown, G.R.; Maglott, D.R. NCBI reference sequences (RefSeq): Current status, new features and genome annotation policy. Nucleic Acids Res. 2012, 40, D130-D135. [CrossRef] [PubMed]

15. Boratyn, G.M.; Camacho, C.; Cooper, P.S.; Coulouris, G.; Fong, A.; Ma, N.; Madden, T.L.; Matten, W.T.; McGinnis, S.D.; Merezhuk, Y.; et al. BLAST: A more efficient report with usability improvements. Nucleic Acids Res. 2013, 41, W29-W33. [CrossRef] [PubMed]

16. Stothard, P. The sequence manipulation suite: JavaScript programs for analyzing and formatting protein and DNA sequences. BioTechniques 2000, 28, 1102-1104. [PubMed]

17. Artimo, P.; Jonnalagedda, M.; Arnold, K.; Baratin, D.; Csardi, G.; de Castro, E.; Duvaud, S.; Flegel, V.; Fortier, A.; Gasteiger, E.; et al. Expasy: SIB bioinformatics resource portal. Nucleic Acids Res. 2012, 40, W597-W603. [CrossRef] [PubMed]

18. Schmitt-Engel, C.; Schultheis, D.; Schwirz, J.; Strohlein, N.; Troelenberg, N.; Majumdar, U.; Dao, V.A.; Grossmann, D.; Richter, T.; Tech, M.; et al. The iBeetle large-scale RNAi screen reveals gene functions for insect development and physiology. Nat. Commun. 2015. [CrossRef] [PubMed]

19. Whangbo, J.S.; Hunter, C.P. Environmental RNA interference. Trends Genet. 2008, 24, 297-305. [CrossRef] [PubMed]

20. Huang, G.Z.; Allen, R.; Davis, E.L.; Baum, T.J.; Hussey, R.S. Engineering broad root-knot resistance in transgenic plants by RNAi silencing of a conserved and essential root-knot nematode parasitism gene. Proc. Natl. Acad. Sci. USA 2006, 103, 14302-14306. [CrossRef] [PubMed]

21. Yadav, B.C.; Veluthambi, K.; Subramaniam, K. Host-generated double stranded RNA induces RNAi in plant-parasitic nematodes and protects the host from infection. Mol. Biochem. Parasitol. 2006, 148, $219-222$. [CrossRef] [PubMed]

22. Mao, Y.B.; Cai, W.J.; Wang, J.W.; Hong, G.J.; Tao, X.Y.; Wang, L.J.; Huang, Y.P.; Chen, X.Y. Silencing a cotton bollworm p450 monooxygenase gene by plant-mediated RNAi impairs larval tolerance of gossypol. Nat. Biotechnol. 2007, 25, 1307-1313. [CrossRef] [PubMed]

23. Ahmad, A.; Negri, I.; Oliveira, W.; Brown, C.; Asiimwe, P.; Sammons, B.; Horak, M.; Jiang, C.; Carson, D. Transportable data from non-target arthropod field studies for the environmental risk assessment of genetically modified maize expressing an insecticidal double-stranded RNA. Transgenic Res. 2016, 25, 1-17. [CrossRef] [PubMed]

24. Lundgren, J.G.; Huber, A.; Wiedenmann, R.N. Quantification of consumption of corn pollen by the predator Coleomegilla maculata (Coleoptera: Coccinellidae) during anthesis in an Illinois cornfield. Agric. For. Entomol. 2005, 7, 53-60. [CrossRef] 
25. Romeis, J.; Meissle, M.; Alvarez-Alfageme, F.; Bigler, F.; Bohan, D.A.; Devos, Y.; Malone, L.A.; Pons, X.; Rauschen, S. Potential use of an arthropod database to support the non-target risk assessment and monitoring of transgenic plants. Transgenic Res. 2014, 23, 995-1013. [CrossRef] [PubMed]

26. Good, R.T.; Varghese, T.; Golz, J.F.; Russell, D.A.; Papanicolaou, A.; Edwards, O.; Robin, C. Offtarget Finder: A web tool for species-specific RNAi design. Bioinformatics 2016, 32, 1232-1234. [CrossRef] [PubMed] 